\title{
Performance Evaluation of Routing Protocols In Hybrid Network
}

\author{
Manjula $S^{1}$, Suresha $^{2}$ \\ ${ }^{1}$ Research Scholar DoS in Computer Science, University of Mysore Manasagangotri, Mysore, India \\ ${ }^{2}$ Professor, DoS in Computer Science, University of Mysore Manasagangotri, Mysore, India
}

\begin{abstract}
In Mobile Ad hoc Network (MANET) the mobile devices are able to move independently by configuring themselves in the network. The topology is not fixed in MANETs. To increase the coverage area and to access the existing services, MANETs are combined with different networks such as internet to form a hybrid network. To combine different networks, devices such as Gateways are required. For the connection of mobile nodes with internet, those mobile nodes must first find and register with the gateways using gateway discovery mechanism. In our paper, we are using the concept of Dynamic gateways. We are experimenting with several parameters to select gateway out of multiple gateways. In our paper we have proposed a load balancing algorithm which addresses this issue. Our algorithm outperforms when compared with existing algorithms by balancing the load among gateways. We have compared DSDV and DSR with our proposed method which uses AODV routing protocol with additional parameters. The parameters used for the performance analysis of our algorithm are Packet delivery ratio, throughput and Average end-to-end delay.
\end{abstract}

Keywords: MANET, Internet-MANET, Hybrid Network, Routing Protocols, Gateway Discovery, DSDV, DSR.

\section{Introduction}

Ad hoc networks [1] consist of wireless mobile devices that are able to communicate even if they are outside of their radio ranges because the intermediate nodes will route the packets from the source node to the destination node. Originally, the investigation was centered in developing isolated and independent adhoc networks useful to collaborate in certain restricted environments like in the case of natural catastrophes. However, more recently, the attention has been focused in studying heterogeneous networks. The interaction between ad hoc networks and other types of networks, like cellular networks, infrastructure-based WLANs (Wireless Local Area Networks) [2] and, especially, wired networks raises great interest. The communication between wireless ad hoc networks and infrastructure-based networks is essential to extend Internet beyond its traditional scope, to remote inaccessible areas, making Web services in ad-hoc networks available anytime, anywhere. The mobile nodes in a wireless adhoc network must be able to detect available gateways and select one of them if they want to have Internet access. On the other hand, real-time applications have special Quality of Service (QoS) requirements that must be satisfied to function properly and they are expected to maintain their quality level in heterogeneous networks. New gateway discovery mechanisms should be designed thinking over the requirements of real-time flows because since Internet access to mobile nodes is provided through gateways, the quality of such service depends on the selection procedure used by ad-hoc mobile nodes to choose the most convenient gateway and register with. Besides, the intrinsic functioning characteristics of the selected gateway mechanism will influence the service level degree that a particular flow is able to obtain and maintain. A Mobile Ad-hoc Network is a collection mobile nodes which doesn't have any fixed topology. Each node in MANET can move independently in any direction. Because of this links between mobile nodes will change frequently. Each mobile node consists of wireless interface and communicates with other nodes through radio signals. Each mobile node maintains the information required to properly route the packets. MANETs use different routing protocols like AODV, DSDV, DSR etc., to route the traffic between nodes. To widen the MANET Network coverage and application domain, we integrated MANET with Internet forming a Hybrid Network. Mobile nodes in MANET can be connected to the Internet through an interface called gateways. For a mobile node to get connected to the internet it has to first find the available gateways and register with it through gateway discovery mechanism. Once the mobile node gets connected to the gateway it can communicate with the remote node which is connected to the internet. In our paper we are using dynamic gateway for the communication of mobile nodes and fixed nodes. A dynamic gateway is a MANET node with extra capability, which is one hop away from foreign agent. Thus the movement of dynamic gateways is limited to the coverage of foreign agent. When dynamic gateways move out of coverage area of foreign agent it becomes normal MANET node not a dynamic gateway. Figure 1 shows MANETInternet Connectivity.

This integration of MANET-Internet can be simulated using NS2 simulator. In this kind of networks, various issues arise. Two of the issues of interest in the current paper are discussed here. For a mobile node to get connected to gateways, it has to first find and then register with the gateway. This can be achieved in one of three ways: proactive, reactive or hybrid. Second issue in hybrid network is congestion among mobile nodes and gateways. To circumvent this problem, some load balancing mechanism must be employed to reduce packet loss due to congestion.

In our paper we proposed an algorithm which reduces the congestion in the network by balancing the load among dynamic gateways. 


\section{International Journal of Science and Research (IJSR) \\ ISSN (Online): 2319-7064}

Index Copernicus Value (2013): 6.14 | Impact Factor (2014): 5.611

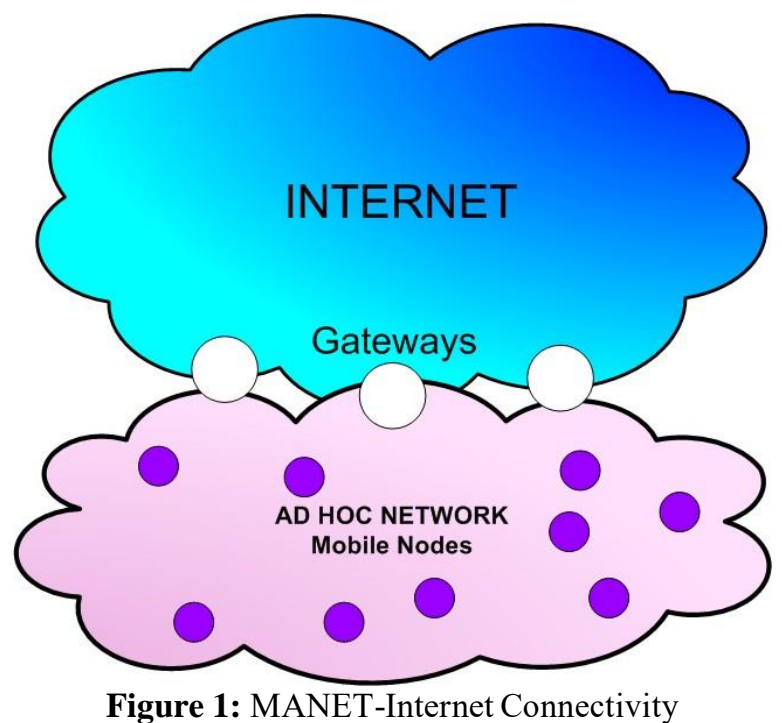

\section{Related Work}

In this section we discuss briefly on the existing work done on integrated Internet-MANET.

- For hybrid networks various architectures are present in the literature. Internet Access for Mobile Ad-hoc Network is described in [4].

- A study on combining MANET with Internet is described in [8].

- In [3] a review of various types of routing protocol such as Proactive, Reactive and Hybrid has been given.

- The traditional gateway discovery mechanisms are described and simulated in [4].

- Study of different types of mobility models are present in the literature. A survey of mobility models simulated in our paper is described in [6].

- The concept of gateway load balancing is addressed by various authors in the literature. In [9], a review of existing gateway load balancing strategies is covered.

- In [10] maximal source coverage algorithm is used to dynamically adjust the proactive zone of the Integrated Internet-MANET. This was the first

- attempt to address the issue of adaptive gateway discovery.

- The issue of path load balanced routing was discussed and presented in [11]. In [12], an optimized version of the approach presented in [11] was discussed.

- In [13], a novel method of adaptive gateway discovery was discussed which worked in a distributed mode.

- In [10] and [13], adaptive gateway discovery mechanisms were presented which used the fuzzy logic concepts to adapt the gateway discovery according to various parameters.

- The concept of dynamic gateways is studied and implemented in [13].

\section{Routing Protocols}

A routing protocol indicates how routers in a network communicate with each other and report changes. It uses software and algorithms to determine optimal path and perform data transfer between network nodes. MANET routing protocols are classified into 3 types:

\section{Proactive}

Proactive routing protocols are also called as Table Driven routing protocol because they have to maintain routing information of the nodes in a table. Each node in proactive routing protocol maintains such table containing routing information so that data can be transferred to the destination. Each entry in the table contains information such as cost of the route to be followed and next hop to reach a node. Proactive routing protocols are not recommended for large networks, since it is difficult to maintain table entries for large network. Example of proactive protocol is DSDV (Destination Sequenced Distance Vector).

\section{Reactive}

Reactive routing protocols are also called as On Demand routing protocols because in reactive routing protocol routes are established only on demand. In this protocol when a source wants to communicate with the destination, it starts a route discovery mechanism to find an optimal path to the required destination. Here, the routes are created on demand by broadcasting Route Request packets. Example of Reactive protocol is AODV (Ad hoc on demand Distance Vector), AOMDV (Ad hoc on demand Multipath Distance Vector), DSR (Dynamic Source Routing) etc.

\section{Hybrid}

Hybrid routing protocol is a combination of both Proactive and Reactive routing protocol. Hybrid protocol was proposed to reduce the routing overhead caused by proactive protocol and also to reduce the latency caused by route discovery in reactive protocol. Example of hybrid protocol is ZRP (Zone Routing Protocol) and TORA (Temporarily Ordered Routing Algorithm).

\section{Gateway Discovery}

A Mobile node which wants to communicate with a wired node which is connected to an internet first needs to find the available gateways through gateway discovery mechanism and then gets connected to it. There are different ways to facilitate this interconnection, which are discussed in the literature [2]. There are three type of gateway discoveries which are briefly explained below.

\section{Proactive Gateway Discovery}

In this approach, the gateway discovery mechanism is initiated by the gateway itself by periodically broadcasting gateway advertisement messages (GW_ADV). The advertisement intervals should be set in such a way that network is not flooded with unnecessary GW_ADV message. Only those nodes within the transmission range of gateway receive the GW_ADV message. And the nodes outside the transmission range of gateway do not receive GW_ADV message. A mobile node which wants to communicate with the wired node which is connected to the internet sends a reply to the GW_ADV message and gets connected to the gateway. All the traffic from the source node is routed to the destination through gateway to which it is connected. 


\section{International Journal of Science and Research (IJSR) \\ ISSN (Online): 2319-7064 \\ Index Copernicus Value (2013): 6.14 | Impact Factor (2014): 5.611}

\section{Reactive Gateway Discovery}

This approach is the opposite of Proactive gateway discovery. GW_ADV messages are not periodically broadcasted in Reactive gateway discovery. Here the mobile node which wants to communicate with the internet initiates gateway discovery mechanism by broadcasting a gateway solicitation (GW_SOL) message. When a gateway receives this GW_SOL message it replies with GW_ADV message to the requesting mobile node.

\section{Hybrid Gateway Discovery}

This gateway discovery process combines both Proactive and Reactive gateway discovery forming a Hybrid gateway discovery Mechanism. To reduce the disadvantage of Proactive/Reactive Gateway Discovery both are combined. For mobile nodes within the range of gateway, proactive gateway discovery is used and for nodes outside the range of gateway, reactive gateway discovery is used[5].

\section{Gateway Selection Schemes}

In the MANET to the Internet connectivity, the communication with external host requires the use of a MANET node acting as the Gateway that is not controlled by the telecommunication operator and the gateway can freely move. Single gateway or multi- gateway is supported and must be selected carefully since the gateway is end of MANET route to the internet. The method of selecting the node to operate as gateway as well as the treatment associated to its mobility is the main characteristics of this mechanism. The list of gateway selection schemes for MANET to Internet connectivity that was collected from several researches based on topics and mechanisms used during years from 2004 to present is considered. Various selection schemes are
A. Hop Count Based Schemes
B. Gateway Load Balancing Based Scheme
C. Secured Gateway Based Scheme
D. Path Quality Based Scheme
E. Path Load Based Scheme
F. Multi-Metrics Based schemes

\section{Existing System}

\begin{abstract}
A. Destination-Sequenced Distance-Vector Routing (DSDV) is a table-driven routing scheme for ad hoc mobile networks based on the Bellman-Ford algorithm. It was developed by C. Perkins and P.Bhagwat in 1994. The main contribution of the algorithm was to solve the routing loop problem. Each entry in the routing table contains a sequence number, the sequence numbers are generally even if a link is present; else, an odd number is used. The number is generated by the destination, and the emitter needs to send out the next update with this number. Routing information is distributed between nodes by sending full dumps infrequently and smaller incremental updates more frequently.
\end{abstract}

If a router receives new information, then it uses the latest sequence number. If the sequence number is the same as the one already in the table, the route with the better metric is used. Stale entries are those entries that have not been updated for a while. Such entries as well as the routes using those nodes as next hops are deleted.

Disadvantages of existing system:

- Always gateway node selection will be static.

- Not energy efficient.

- Not reliabile.

DSDV requires a regular update of its routing tables, which uses up battery power and a small amount of bandwidth even when the network is idle. Whenever the topology of the network changes, a new sequence number is necessary before the network re-converges; thus, DSDV is not suitable for highly dynamic or large scale networks. (As in all distance-vector protocols, this does not perturb traffic in regions of the network that are not concerned by the topology change.)

The disadvantage of this protocol is that the route maintenance mechanism does not locally repair a broken link. Stale route cache information could also randomly result in inconsistencies during the route reconstruction phase. The connection setup delay is higher than in tabledriven protocols. Even though the protocol performs well in static and low-mobility environments, the performance degrades rapidly with increasing mobility. Also, considerable routing overhead is involved due to the source- routing mechanism employed in DSR. This routing overhead is directly proportional to the path length.

B. Dynamic Source Routing (DSR) is a routing protocol for wireless mesh networks. It is similar to AODV in that it forms a route on-demand when a transmitting node requests one. However, it uses source routing instead of relying on the routing table at each intermediate device. Determining source routes requires accumulating the address of each device between the source and destination during route discovery. The accumulated path information is cached by nodes processing the route discovery packets. The learned paths are used to route packets. To accomplish source routing, the routed packets contain the address of each device the packet will traverse. This may result in high overhead for long paths or large addresses, like IPv6. To avoid using source routing, DSR optionally defines a flow id option that allows packets to be forwarded on a hop-by-hop basis.

This protocol is truly based on source routing whereby all the routing information is maintained (continually updated) at mobile nodes. It has only two major phases, which are Route Discovery and Route Maintenance. Route Reply would only be generated if the message has reached the intended destination node (route record which is initially contained in Route Request would be inserted into the Route Reply).

To return the Route Reply, the destination node must have a route to the source node. If the route is in the Destination Node's route cache, the route would be used. Otherwise, the node will reverse the route based on the route record in the Route Request message header (this requires that all links are symmetric). In the event of fatal transmission, the Route Maintenance Phase is initiated whereby the Route Error packets are generated at a node. The erroneous hop will be removed from the node's route cache; all routes containing 


\section{International Journal of Science and Research (IJSR) \\ ISSN (Online): 2319-7064}

Index Copernicus Value (2013): 6.14 | Impact Factor (2014): 5.611

the hop are truncated at that point. Again, the Route Discovery Phase is initiated to determine the most viable route.

\section{Proposed System}

In our proposed system we have considered set of 18 mobile nodes, starting from initial position to respective position in the network.

The steps followed in our algorithm are :

Step 1.Initially all the mobile nodes are in static position in two groups.

Step 2.After some time they start to move randomly taking various positions. There is one internet host which is static.

Step 3. Distance to various mobile nodes from static host is calculated, that is one of the parameter to identify mobile node as a gateway.

Step4.Other added parameter are there to consider a particular mobile nodes as a gateway.

Step 5.The mobile nodes having high energy and highest packet delivery ratio and least distance from static wired host are identified as gateways.

Step 6.After the selection of the gateways depending upon these parameters, data transmission takes place between mobile nodes and gateways.

Step 7. After sometime transmission of data takes place between gateways and internet host.

Step 8. In this way communication between normal mobile nodes, gateways and internet host takes place.

The parameters used for selecting mobile nodes as gateways.

1. Packet Delivery Ratio : the ratio of the number of delivered data packet to the destination to the number of packets sent. This illustrates the level of delivered data to the destination which is given by the expression:

Number of packets received / $\sum$ Number of packets sent [1] The greater value of packet delivery ratio means the better performance of the protocol.

2. Minimum Hop Distance: The Euclidean distance between points $\mathrm{p}$ and $\mathrm{q}$ is the length of the line segment connecting them. In Cartesian coordinates, if $\mathrm{p}=\left(p_{1}, p_{2}, \ldots, p_{n}\right)$ and $\mathrm{q}=$ $\left(q_{1}, q_{2}, \ldots, q_{n}\right)$ are two points in Euclidean n-space, then the distance (d) from $\mathrm{p}$ to $\mathrm{q}$, or from $\mathrm{q}$ to $\mathrm{p}$ is given by $d(p, q)$.Very often, especially when measuring the distance in the plane, we use the formula for the Euclidean distance. According to the Euclidean distance formula, the distance between two points in the plane with coordinates $(x, y)$ and $(a, b)$ is given by $\operatorname{dist}((x, y),(a, b))=\sqrt{ }(x-a)^{2}+(y-b)^{2}$ [2] The position of a point in a Euclidean $n$-space is a Euclidean vector. So, $\mathrm{p}$ and $\mathrm{q}$ are Euclidean vectors, starting from the origin of the space, and their tips indicate two points. The Euclidean norm, or Euclidean length, or magnitude of a vector measures the length of the vector.

A vector can be described as a directed line segment from the origin of the Euclidean space (vector tail), to a point in that space (vector tip). If we consider that its length is actually the distance from its tail to its tip, it becomes clear that the Euclidean norm of a vector is just a special case of Euclidean distance: the Euclidean distance between its tail and its tip. The distance between points $\mathrm{p}$ and $\mathrm{q}$ may have a direction (e.g. from $\mathrm{p}$ to $\mathrm{q}$ ), so it may be represented by another vector.In a three-dimensional space $(n=3)$, this is an arrow from $\mathrm{p}$ to $\mathrm{q}$, which can be also regarded as the position of $\mathrm{q}$ relative to $\mathrm{p}$. It may be also called a displacement vector if $\mathrm{p}$ and $\mathrm{q}$ represent two positions of the same point at two successive instants of time.

The Euclidean distance between $\mathrm{p}$ and $\mathrm{q}$ is just the Euclidean length of this distance (or displacement) vector.

3. High Residual Energy: The energy model represents the energy level of nodes in the network. The energy model defined in a node has an initial value that is the level of energy the node has at the beginning of the simulation. This energy is termed as initial Energy. In simulation, the variable "energy" represents the energy level in a node at any specified time. The value of initial energy is passed as an input argument. A node looses a particular amount of energy for every packet transmitted and every packet received. As a result, the value of initial energy_ in a node gets decreased. The current value of energy in a node after receiving or transmitting routing packets is the residual energy. Data Transmission is established between nodes using UDP agent and CBR traffic. Residual energy of the node is evaluated by accessing inbuilt variable "energy" to find energy procedure at different times. Data transmission from mobile nodes to internet through gateway happens. Proposed protocol takes care of configuration of dynamic gateway selection.

Advantages of Proposed system:

- Always gateway node selection will be dynamic.

- Energy efficient.

- Reliable

\section{Simulation Setup}

This Simulation has been implemented using Network Simulator 2 (ns-2.33). The Simulation consists of 18 mobile nodes, 1 fixed node, 2 gateways. The simulation area is of $1000 \mathrm{~m}$ length $* 1000 \mathrm{~m}$ width. The bandwidth of all the fixed links are set to $5 \mathrm{Mbps}$. The radio range of each wireless transmitter is set to $250 \mathrm{~m}$. All the simulations were run for a total of 500 seconds. The traffic sources used in this simulation is CBR sending data packet with size 512 bytes at an interval of 0.25 seconds. Table 1 shows the general parameters used in simulation.

Table 1: General parameters used in Simulation

\begin{tabular}{|c|c|}
\hline Parameter & Value \\
\hline Simulation Area & $1000 \mathrm{~m}$ X 1000m \\
\hline MAC Protocol & IEEE 802.11 \\
\hline Number of Mobile Nodes & 18 \\
\hline Number of Gateways & 2 \\
\hline Number of Fixed nodes & 1 \\
\hline Antenna Type & Two Ray Ground t \\
\hline Propagation Model & Omni antenna \\
\hline Number of Connections & 5,10 \\
\hline Packet Size & 512 byte \\
\hline Routing Protocols & DSDV, DSR \& DYNGWAY \\
\hline Traffic Sources & CBR \\
\hline Simulation Time & 300 Sec. \\
\hline Mobility Model & Random waypoint \\
\hline Pause Time & 10 sec \\
\hline
\end{tabular}




\section{International Journal of Science and Research (IJSR) \\ ISSN (Online): 2319-7064}

Index Copernicus Value (2013): 6.14 | Impact Factor (2014): 5.611

The performance of our algorithm is compared with existing strategies like DSDV and DSR in integrated InternetMANET. The output shows that our proposed algorithm performs better compared to DSDV and DSR routing algorithms. Figure 2 shows a Snapshot of the simulation performed.

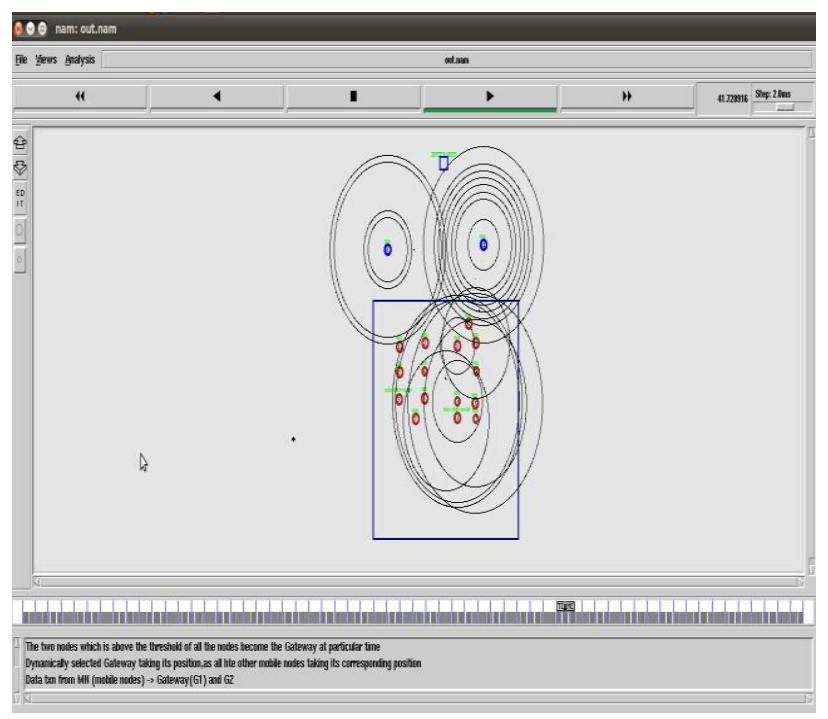

Figure 2: Simulation Snapshot

\section{Performance Analysis}

The Performance of different routing protocols are compared with our protocol.

\section{Packet Delivery Ratio}

Packet delivery ratio is the ratio of packet that has been successfully received by the destination node to the number of packets that have been transmitted by the source. Higher the packet delivery ratio better is the performance. Figure 3 shows that the performance of our proposed algorithm is better than the existing strategies. Hence the performance of Random Direction model is better in terms of Packet delivery Ratio. The Packet Delivery Ratio is calculated using the formula:

$$
F=\frac{1}{C} \sum_{f=1}^{C} \frac{R_{f}}{T_{f}}
$$

$F$ : Fraction of successfully delivered packets. $C$ : Total number of flows. $f$ : Unique flow id. $R f$ : Count of unique packets received from flow $f$. Tf : Count of packets ransmitted to flow $f$.

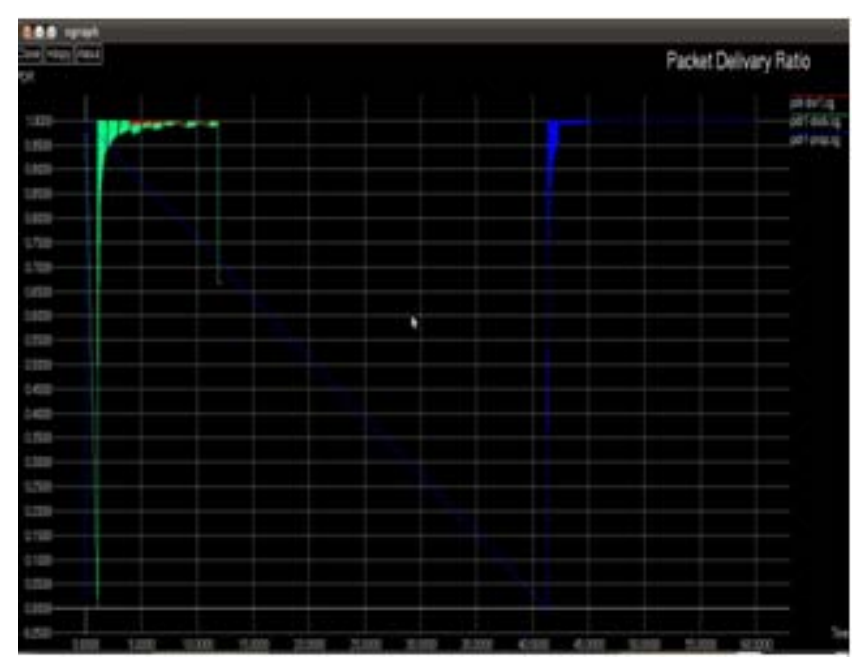

Figure 3: Packet Delivery Ratio

\section{Throughput}

Throughput is the amount of packets that are successfully delivered to the destination in a given time period. It is represented in bps. Higher throughput indicates better performance. Throughput is calculated using the formula:

\section{Throughput $=$ received_data $* 8 /$ DataTransmissionPeriod .} [4] Figure 4 shows that the performance of our proposed strategy is better than the existing strategies.

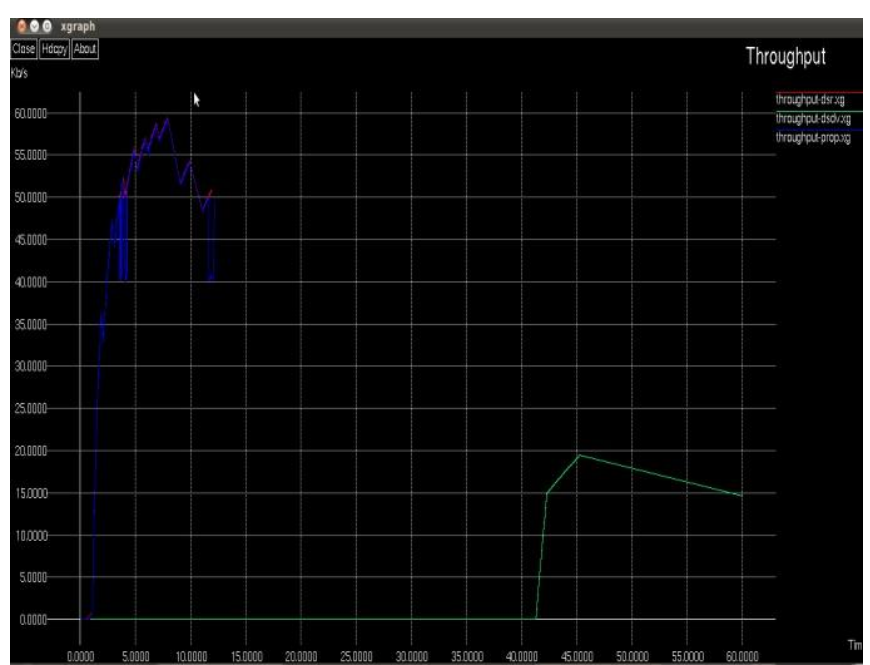

Figure 4: Throughput

3. Average End-to-End Delay: It is defined as the average time taken by the data packets to propagate from source to destination across a MANET. This includes all possible delays caused by buffering during routing discovery latency, queuing at the interface queue, and retransmission delays at the MAC, propagation and transfer times. Figure 5 shows that the performance of our proposed algorithm is better than the existing strategies 


\section{International Journal of Science and Research (IJSR) \\ ISSN (Online): 2319-7064}

Index Copernicus Value (2013): 6.14 | Impact Factor (2014): 5.611

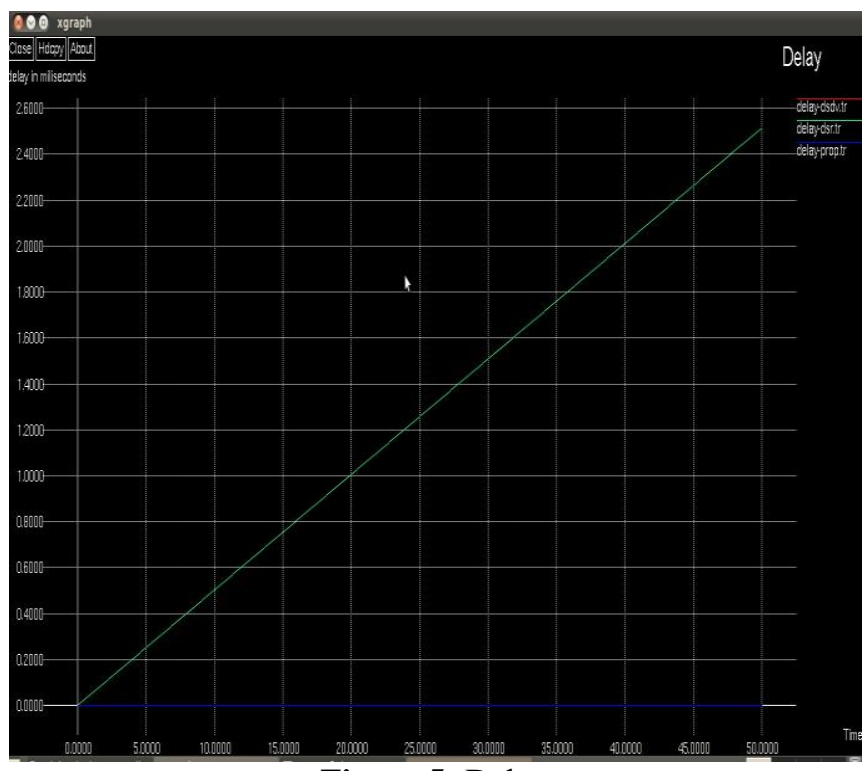

Figure 5: Delay

\section{Conclusion}

In This paper we have created a simulation of hybrid network which is composed of Internet and MANET. We have compared the performance of proposed protocol with DSDV and DSR routing protocols. Our simulation result shows that our proposed algorithm performs better than existing approaches in terms of packet delivery ratio, throughput and average delay. In the future, we intend to find methods to perform service discovery and efficient usage of those services which are present in different networks with scalability.

\section{References}

[1] S. Basagni, M. Conti, S. Giordando and I. Stojmenovic, Mobile Ad Hoc Networking, IEEE Press \& Wiley Inter-Science, 2004.

[2] D. Cavalcanti, C. Cordeiro, D. Agrawal, B. Xie and A.Kumar, "Issues in Integrating Cellular Networks, WLANs, and MANETs: A Futuristic Heterogeneous Wireless Network", IEEE Wireless Communications Magazine, Vol. 12, No. 3, pp. 30-4, April 2005.

[3] A. Sharmila, R. Selvakumar, M. Preetha "A Study Of MANET Integration Through Internet", IJCSMC, Vol. 2, Issue. 7, July 2013

[4] Ali Hamidian, Ulf Korner and Anders Nilsson "Performance Of Internet Access Solutions In Mobile Ad hoc Networks", Springer's Lecture Notes in Computer Science (LNCS), pp 189-201, 2004.

[5] Harjeet Kaur, Varsha Sahni and Dr. Manju Bala "A Survey of Reactive, Proactive and Hybrid Routing Protocols in MANET: A Review”, IJCSIT, Vol. 4 (3), 2013.

[6] Tracy Camp, Jeff Boleng and Vanessa Davies "A Survey of Mobility Models for Ad Hoc Network Research", WCMC, vol. 2, no. 5, pp. 483-502, 2002.

[7] "The Network Simulator: Contributed Code": http://nsnam.isi.edu/nsnam/index.php/Contributed_Cod e.

[8] A. Hamidian. "A Study of Internet Connectivity for Mobile Ad Hoc Networks in NS 2".Master's thesis.
Department of Communication Systems, Lund Institute of Technology, Lund University. January 2003.

[9] Rafi U Zaman, Khaleel Ur Rahman Khan and A. Venugopal Reddy, "A Review of Gateway Load Balancing Strategies in Integrated Internet-MANET", International Workshop on Advances in Peer-Peer Technology IWAP2PT'09 co-located with IMSAA-09, IIIT Bangalore, 9-11 December 2009. Proceedings in IEEE Digital Library, pages: $141-146$.

[10] Pedro M. Ruiz, Antonio F. Gomez-Skarmeta: Maximal Source Coverage Adaptive Gateway Discovery for Hybrid Ad Hoc Networks, Lecture Notes in Computer Science, vol.3158, 28-41, (2004).

[11] Khan K. U. R., Reddy A. V., Zaman R. U., Kumar M. "An Effective Gateway Discovery Mechanism in an Integrated Internet-MANET (IIM)," Proc. of the International Conference on Advances in Computer Engineering, India, pp. 24-28, June 2010.

[12]Kumar, R., Misra, M. and Sarje, A.K., "An Efficient Gateway Discovery in Ad Hoc Networks for Internet Connectivity", Proc. Of the International Conference on Computational Intelligence and Multimedia Applications, pp 275-281, 2007.

[13] Javaid, U., Rasheed, T.M., Meddour, D., Ahmed, T.: Adaptive Distributed Gateway Discovery in Hybrid Wireless Networks. WCNC - 2008, 2735-2740. (2008). 